\title{
Relaciones intergeneracionales: profesoras seniors con niños y niñas del Programa Socioeducativo, Penco, Chile
}

\author{
Carolina Soledad Monsalve-Reyes ${ }^{1}, \mathrm{M}^{\mathrm{a}}$ Angélica Parada ${ }^{2}$, \\ Natali Ormeño Stuardo ${ }^{3}$, Verónica Gómez ${ }^{4}$
}

\begin{abstract}
RESUMEN
Este artículo da cuenta de la investigación social efectuada en la comuna de Penco, Chile, que describe la valoración atribuida por los actores a las relaciones intergeneracionales, en el marco de la implementación del programa de refuerzo socioeducativo llevado a cabo por profesoras seniors de la casa del adulto mayor a niños y niñas de la misma comuna.

La metodología es cualitativa, con un enfoque fenomenográfico, ya que se busca comprender, mediante el mismo relato de los protagonistas, los aspectos que destacan de la valoración o importancia atribuida a la experiencia y sus resultados.
\end{abstract}

Palabras claves: intergeneracional, personas mayores, niñas y niños, socioeducación

\section{Intergenerational Relations: senior teachers with children in the Socio-Educational Program of Penco, Chile}

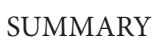

This article reports on social research conducted in Penco District, Chile, describing the valuation ascribed by stakeholders to intergenerational relations, within the framework of the socio-educational program implemented by senior teachers at the local home for older people, for children in the same district.

1 Gerontóloga Social, académica de Trabajo Social, Departamento de Ciencias Sociales, Facultad de Comunicación, Historia y Ciencias Sociales, Universidad Católica de la Santísima Concepción, Chile. E-mail: carolinamonsalve@ucsc.cl

2 Trabajadora Social, Alumnni UCSC. E-mail: mparadasbarros@gmail.com Trabajadora Social, Alumnni UCSC. E-mail: ormenostuardo.natali@gmail.com Académica de Trabajo Social, Departamento de Ciencias Sociales, Facultad de Comunicación, Historia y Ciencias Sociales, Universidad Católica de La Santísima Concepción, Chile.E-mail: vegomez@ucsc.cl 
Relaciones intergeneracionales: profesoras seniors con niños y niñas del Programa Socioeducativo, Penco, Chile /Monsalve-Reyes et al..

The methodology used is qualitative, built on a phenomenographic approach, with the goal of garnering an understanding of key elements in the valuation or importance attributed to the experience and its results, drawn from the narrative of the protagonists in the process.

Keywords: intergenerational, older people, children, socio-education

\section{Relações intergeracionais: professoras sêniores com crianças do Programa Socioeducativo, Penco, Chile}

RESUMO

Este artigo apresenta a investigação social efetuada no município de Penco, no Chile, que descreve a valorização atribuída pelos atores às relações intergeracionais, no âmbito da implementação do programa de reforço socioeducativo realizado por professoras sêniores da casa do idoso para crianças do mesmo município.

A metodologia é qualitativa, com um foco fenomenográfico, uma vez que se procura compreender, através do mesmo relato dos protagonistas, os aspectos que destacam da valorização ou importância atribuída à experiência e seus resultados.

Palavras-chave: intergeracional, idosos, crianças, socioeducação

\section{Introducción}

El envejecimiento paulatino de la población a nivel mundial es un fenómeno que se envidencia día a día. Según la CEPAL, esta "población aumentará a un ritmo de 3,5\% entre el período de 2020 a 2025, ocurriendo esto tres veces más rápido que el índice de la población total" (CEPAL, 2002). Existe un precedente que indica que para el año 2050 se proyecta "que en la mayoría de los países latinoamericanos la población de adultos mayores superará el 30\%, en tanto en nuesto país ocurrirá que para el año 2050 un 23,5\% de la población corresponderá a mayores de 60 años" (CEPAL, 2002).

Los antecedentes de dicho fenómeno demográfico en Chile se plasman en la cuarta encuesta Nacional de Inclusión y Exclusión, elaborada en 2015 por el Servicio Nacional del Adulto Mayor, SENAMA, en la que se señala los siguientes indicadores de crecimiento demográfico, referente a los quinquenios 2005-2010, vislumbrando que: 
Para el 2010, esta cifra se ubicaría en 61,5 personas de 60 años y más cada 100 menores de 15 años y se espera que alrededor del año 2025 la población de mayores supere a la de los niños, llegando a 116,3 personas mayores por cada 100 personas entre 0 y 14 años para ese año. (SENAMA, 2015).

De lo anterior es posible señalar además que Chile se encuentra entre los países que presenta mayor índice de envejecimiento, por lo que es de vital importancia generar mayores espacios que incluyan y beneficien a esta población, siendo necesario abordar el envejecimiento desde focos y miradas integradoras en todo el país.

Teniendo como referencia que Chile se rige mediante un Estado subsidiario, en el que las políticas sociales se enfocan principalmente en los grupos vulnerables, ya sea pobreza o extrema pobreza, en la praxis, es el Estado el que debe adquirir un rol activo en la formulación de políticas públicas que involucren aspectos sociales, sanitarios y económicos con base en el fenómeno demográfico del envejecimiento, con el fin de que las personas mayores puedan desenvolverse de manera íntegra, entendiendo que "el punto de partida no es la existencia de personas con necesidades, que deben ser asistidas, sino sujetos con derechos que obligan al Estado y al resto de la sociedad" (CEPAL, 2009). Siendo así, es trascendental que la entidad gubernamental se involucre en la formulación de leyes dirigidas a la población mayor, que brinden protección y subsanen necesidades que presente dicha población.

Para alcanzar nuestro objetivo de una sociedad más cohesiva, necesitamos lanzar un diálogo en todos los niveles - local, nacional e internacional- que implique a todas las generaciones y los grupos sociales. Debemos encontrar un nuevo equilibrio y nuevas relaciones entre las generaciones mediante enfoques innovadores y políticas que promuevan la solidaridad intergeneracional por una sociedad cohesiva y sostenible. El desarrollo social sostenible será posible sólo con el contacto genuino y la coexistencia entre las generaciones cuya base es la solidaridad intergeneracional. (Sedmak \& Parent, 2008) 
Relaciones intergeneracionales: profesoras seniors con niños y niñas del Programa Socioeducativo, Penco, Chile /Monsalve-Reyes et al..

Es incuestionable el potencial productivo, fundamentalmente en el nivel psicosociológico, de estos adultos mayores, con gran valor y experiencia, por eso la importancia concedida a los contactos sociales formales e informales, ya sea en redes interactivas o interacciones puntuales, insertas en programas intergeneracionales o mediante la potenciación de participaciones activas de los mayores en la comunidad (Moral, 2017).

A la edad se le ha dado un carácter fijo, de ahí que la existencia de conflictos, la sensación de amenaza o los estereotipos de comportamiento extendidos sobre los niños, niñas, jóvenes y los ancianos graviten en los análisis intergeneracionales (Acosta \& Martín, 2017).

Los programas intergeneracionales constituyen una metodología de acción social que actúa en la comunidad sobre las bases de un modelo de solidaridad intergeneracional (Pinazo, 2012, citado en Moral, 2017).

Las relaciones entre niños, niñas, jóvenes y mayores, que comúnmente establecíamos y que también se han profundizado en las investigaciones, no es fácil establecerlas fuera de este entorno, pues las percepciones y las actuaciones suelen ser distintas en ambos grupos (Delgado Acosta \& Calero Martín, 2017).

No obstante, las relaciones intergeneracionales se pueden brindar en programas intergeneracionales de una forma más intencionada, lo que no condiciona que se sigan dando de forma natural, aunque la permanencia de las relaciones es la que debe condicionar el programa, para que no sea una experiencia con término breve.

Parece más que razonable pensar que el desarrollo social y comunitario, es decir, la mejora de la vida en sociedad, pasa por superar estas situaciones de fragmentación y distanciamiento debido al debilitamiento de vínculos y por conseguir una mayor cohesión social. Todas aquellas formulas que, como los programas intergeneracionales, promueven el reconocimiento 
de la diversidad y la integración de las perspectivas, capacidades y activos de distintas generaciones, favoreciendo el diálogo y la cooperación entre los diferentes grupos de edad, contribuyen a conseguir dicha cohesión. (Programas Intergeneracionales Bizkaia, 2015)

Mientras las personas envejecen y cada vez más perciben el tiempo como finito, ponen énfasis más en el significado emocional de su vida que en aquellos objetivos que expanden sus horizontes pero que se logran con la carga emocional (Chen \& Jordan, 2016).

La seriedad y la apertura del anciano se combinan con curiosidad y comprensión de los más jóvenes en una mezcla viva dinámica lingüística, que se divide intersubjetivamente (Ehert, 2016).

No existen suficientes oportunidades de encuentro para que las personas mayores y los niños, niñas y jóvenes puedan llevar a cabo proyectos conjuntos; por ello, las políticas sociales deben contener elementos orientados a apoyar iniciativas de fortalecimiento de las relaciones intergeneracionales (Trujillo, Morales \& Arregoces, 2017).

Algunos enfoques podrían ser: intercambios de conocimientos y habilidades entre jóvenes y mayores; asistencia mutua ("los jóvenes ayudan a los ancianos - los ancianos ayudan a los jóvenes"; hogar o asistencia similar para personas mayores proporcionada por jóvenes; personas mayores actuando como mentores para estudiantes escolares etc.), o actividades comunes que involucran ciertos problemas sociales (Juntos Ayer, Hoy y Mañana, 2009).

Romper el aislamiento generacional, así como dar oportunidad de voluntariado a todas las edades, construyendo redes de apoyo social en la comunidad, constituye un apremiante reto. De este modo, la potenciación del intercambio entre las generaciones en la comunidad contribuye a la reducción de la visión negativista sobre el proceso de envejecimiento, favoreciendo el entendimiento y el progreso social, así como a convertirse en una herramienta de reivindicación y transformación social y apoyo a la ciudadanía. 
Relaciones intergeneracionales: profesoras seniors con niños y niñas del Programa Socioeducativo, Penco, Chile /Monsalve-Reyes et al..

Para cada individuo que participa de ellos representan proyectos motivados por eldeseo de compartir esfuerzos e ilusiones (Moral, 2017). En las políticas sociales actuales podemos destacar esta situación como prioritaria de revisar respecto de las relaciones y programas intergeneracionales, en los extremos del ciclo vital.

Es imprescindible el cambio de paradigma; para ello se requeriría entender el concepto de "relaciones intergeneracionales", relaciones que se establecen entre individuos de diferentes cohortes de edado generaciones (Acosta \& Martín, 2017).

En este punto ampliaremos los conceptos a programas intergeneracionales, cuando une a varias generaciones en actividades planificadas con objeto de desarrollar nuevas relaciones y alcanzar objetivos específicos, permitiendo beneficiarse y aprender los unos de los otros (Acosta \& Martín, 2017). En el caso de esta investigación, el programa intergeneracional de refuerzo socioeducativo genera un intercambio mutuo de beneficios, partiendo con el refuerzo para aquellos niños que se encuentran en desventaja en su entorno sociofamiliar, constituyendo una metodología de acción social que actúa en la comunidad, sobre las bases de un modelo de solidaridad intergeneracional (Pinazo, 2012, citado en Moral, 2017).

La Convención sobre los Derechos del Niño reconoce que los padres, así como las familias y las comunidades, "tienen la responsabilidad primordial de la crianza y el desarrollo del niño", y "los Estados Partes prestarán la asistencia apropiada a los padres y a los representantes legales para el desempeño de sus funciones en lo que respecta a la crianza del niño y velarán por la creación de instituciones, instalaciones y servicios para el cuidado de los niños" (Convención sobre los derechos del Niño/a, art. 18). Sin embargo, en algunos contextos de mayor precariedad y vulnerabilidad, la capacidad de las comunidades y de los padres suele verse amenazada a medida que los niños y niñas enfrentan complejos riesgos de protección en su entorno social, diversos conflictos con el sistema escolar formal y otros derivados de la desintegración y 
el estrés familiar. Para mitigar esos riesgos, es importante la participación activa de las comunidades en la protección de los niños y niñas. El aporte de las personas mayores en este sentido, debido a su experiencia y disponibilidad, es un factor esencial a considerar.

El objetivo de esta investigación fue describir la valoración atribuida a las relaciones intergeneracionales en una experiencia entre personas mayores y niños y niñas, por parte de los y las participantes involucrados en el programa intergeneracional de refuerzo socioeducativo realizado por profesoras seniors de la Casa del Adulto Mayor a niños y niños, niñas en la comuna de Penco, Chile.

\section{Metodología}

La metodología utilizada corresponde a una cualitativa, con un enfoque fenomenográfico (González-Ugalde, 2014), fuente primaria, transversal en el segundo semestre de 2016, en la que se efectuó, en la primera parte, entrevistas semiestructuradas a cinco profesoras seniors, cuyas edades fluctuaban entre los 70 y 80 años de edad, a seis adultos responsables de los niños/as de edades entre los 30 y 45 años. De forma posterior se realizó una entrevista grupal a las asesoras seniors, enfocada en profundizar y reflexionar sobre su entrevista individual, y entrevistas a través de dibujos y técnicas lúdicas a cuatro niños y dos niñas entre los 8 y 9 años de edad. Las técnicas de recolección de datos tienen auditabilidad: "las conclusiones surgirán del tipo de datos utilizados y sí podrán ser objeto de auditoria por aquellas personas que quieran evaluar la calidad de la investigación" (Vasilachis, 2006).

\section{Resultados}

Los resultados de la investigación han sido distribuidos por actor involucrado, para así poseer una visión macro de la labor realizada, teniendo que:

Las profesoras seniors destacan poseer un envejecimiento activo, gratificación con la labor realizada, fomento de las relaciones in- 
Relaciones intergeneracionales: profesoras seniors con niños y niñas del Programa Socioeducativo, Penco, Chile /Monsalve-Reyes et al..

tergeneracionales y vinculación con el medio social, dada la particularidad y distribución geográfica donde se implementó el reforzamiento con los niños y niñas. Es decir, tenemos personas mayores que desarrollan esta labor en un espacio comunitario, en el que se les identifica un rol con características positivas - no solo para quienes reciben sus actividades - y alcanzan la visibilización en la comunidad en la que están insertas.

En los niños y niñas se destaca que, mediante la construcción cognitiva, pudieron interactuar socialmente con adultos/as mayores. Se obtuvo que, con métodos lúdicos, las profesoras seniors aportaron en la formación académica y personal, facilitando el aprendizaje de los niños y niñas. En la ludificación del aprendizaje, los niños y niñas percibieron un espacio de confianza en sus destrezas y habilidades, pasando a un segundo plano el exitismo y centrados en el proceso cognitivo emocional del "aprender a aprender".

Los adultos responsables resaltan el aumento del rendimiento escolar y cambios conductuales, debido al apoyo individualizado que cada profesora beniors brindó a los niños y niñas; por otra parte, destacan poseer sentimientos de gratitud por la labor realizada, puesto que las adultas mayores poseían experiencia en el área académica e implementaban métodos innovadores.

Por lo que se infiere que es importante contar con más espacios intergeneracionales para lo que nosotros lo definimos como "lugares", tanto espacios públicos como privados, en los que se genere e intencione la convivencia intergeneracional.

Los adultos responsables identificaron la satisfacción de necesidades educativas de los niños y niñas en el ámbito escolar, a través de la relación que se establece con los adultos mayores, los que en su totalidad evidenciaron un mejoramiento escolar en los niños y niñas, mencionando que dicho intercambio mejoró los resultados en la escuela, lo que se asemejaría a lo plasmado por la IMSERSO en 2010, en cuanto a los beneficios que se generan mediante los programas intergeneracionales. Cabe destacar que, según lo expresado por los adultos responsables de los niños y niños, además 
de la existencia de un vínculo pedagógico, que es por el cual se generan este tipo de programas, también se visualiza un nexo afectivo, por lo que dicho intercambio se aboca, además de a las necesidades de cada integrante, a otros aspectos personales.

Un hallazgo es la relación que se da entre ambos grupos etarios, considerando la bidireccionalidad de la satisfacción vital, como así también la contención emocional entre estas generaciones, elementos que no estaban considerados. Las relaciones satisfactorias disminuyen el sentimiento de soledad que experimentan las personas mayores, como así también provoca en los niños una conexión con el otro que no esta en un ámbito territorial y emocional cercano, lo cual amplía el espectro de las relaciones de niños y niñas, disminuyendo las futuras relaciones de edadismo que se generan en otras etapas del ciclo vital.

Generar espacios de libertad en la vinculación es lo que los padres/madres/responsables y adultos han comprendido como la significación para el/la niño niña, más allá de lo académico, tendiendo a elementos de valor para la vida cotidiana, hoy y tal vez en el futuro.

\section{Conclusiones}

Se concluye así en la necesidad de generar más espacios de interacción entre adultos mayores y niñas y niños, con el fin de erradicar estereotipos negativos de la vejez; además, en la importancia de formular políticas públicas que aborden la temática intergeneracional, fomentando el envejecimiento activo, la participación, el empoderamiento, además de proponer y mejorar estándares de calidad de vida en nuestros adultos mayores.

Por otro lado, estos programas tienden a fortalecer la conciencia de las comunidades en cuanto garantes de derechos y de capacidades respecto de la protección de los niños y niñas, fundamentalmente en contextos más desfavorecidos socialmente. Los asesores seniors representan este elemento como una propuesta de política social local. 
Relaciones intergeneracionales: profesoras seniors con niños y niñas del Programa Socioeducativo, Penco, Chile /Monsalve-Reyes et al..

El objetivo es generar espacios de convivencia entre generaciones para crear una sociedad sostenible basada en la equidad, y además eliminar cualquier discriminación o segregación por edad.

Las personas mayores reconocen que estos espacios son muy nutritivos para su bienestar psicológico, que es prioritario para ellas en relación con las proyecciones de otros ámbitos de sus vidas. Estos espacios permiten trabajar las emociones, tanto para disminuir la soledad en las personas mayores y aumentar su sentimiento de valía, como también para ampliar el contexto de la generatividad de niños y niñas.

Estas experiencias son una oportunidad para potenciar una práctica de refuerzo socioeducativo, como también instancias de encuentros a nivel lúdico: una comunidad con más cohesión y solidaridad entre las generaciones, como fue el hallazgo de esta investigación.

Es primordial que las personas que participen de este tipo de programas tengan un sentido ético y conciencia de los derechos de los niños y niñas, lo que implica visualizar cualquier forma de práctica abusiva de parte de las generaciones adultas mayores.

\section{Bibliografía}

Acosta, C. R. D. \& Martín, C. G. C. (2017). Espacios de convivencia y experiencia intergeneracional. Trabajo Social Hoy, (82), $19-40$.

Chen, J. \& Jordan, L. P. (2016). Intergenerational support and life satisfaction of young-, old- and oldest-old adults in China, Aging \& Mental Health. 22(3), 412-420. doi 10.1080/13607863.2016.1261798

Comisión Económica para América Latina y el Caribe, CEPAL. (2002). Los adultos mayores en América Latina y el Caribe. Madrid.

Comisión Económica para América Latina y el Caribe, CEPAL. (2009). Envejecimiento, derechos humanos y políticas públicas. Santiago, Chile.

Decreto 830, Convención sobre los Derechos del Niño, artículo 18, Diario Oficial de la República de Chile, Santiago, Chile, 27 de septiembre de 1990. 
Delgado Acosta, C. \& Calero Martín, C. (2017). Espacios de convivencia y experiencia intergeneracional. Trabajo Social Hoy, (82), 19-40.

Ehert, S. (2016). Dialog und intergenerationelle Entwicklung zwischen Jung und Alt. Stuttgart, Alemania: Symposium Soziolgie \& Psychologie, Kongress Gerontologie und Geriatrie.

González-Ugalde, C. (2014). Investigación fenomenográfica. Revista Internacional de Investigación en Educación, 7(14), 141-158. Chile.

Juntos Ayer, Hoy y Mañana. (2009). Guia para la planificación y aplicación de proyectos intergeneracionales. Proyecto MATES Mainstreaming Intergenerational Solidarity.

Moral Jiménez, M. (2017). Programas intergeneracionales y participación social: la integración de los adultos mayores españoles y latinoamericanos en la comunidad. Universitas Psychologica, 16(1).

Pinto, T. A., Marreel, I. \& Hatton-Yeo, A. (2009). Guía de ideas para la Planificación y Aplicación de Proyectos Intergeneracionales (Guide of Ideas for Planning and Implementing Intergenerational Projects).

Programas Intergeneracionales Bizkaia. (2015). Hacia una sociedad intergeneracional: ¿Cómo impulsar programas para todas las edades? Guía práctica. Diputación Foral de Bizkaia.

Sedmak, M. \& Parent, A. S. (2008). Solidaridad intergeneracional por una sociedad cohesiva y sostenible. Brdo, Eslovenia: Erste Foundation.

Santorelli, G. D., Ready, R. E. \& Mather, M. A. (2018). Percepciones de la emoción y la edad entre los adultos más jóvenes, de mediana edad y mayores, Envejecimiento y Salud Mental, 22(3), 421-429.

Servicio Nacional del Adulto Mayor, SENAMA. (2015). Cuarta Encuesta Nacional de inclusión y exclusión social de las personas mayores en Chile. SENAMA-FACSO-UCH. Chile: Ediciones Servicio Nacional del Adulto Mayor.

Trujillo, S., Morales, S. \& Arregoces, M. (2017). Encuentros y desencuentros: trabajo biográfico con adultos mayores, de jóvenes estudiantes de Psicología. Revista Enfoques, 2(1). DOI: http://dx.doi.org/10.24267/23898798.213

Unión Europea. Government of the Republic of Slovenia. (2008). Project "Barnahus/Children's house". Recuperado de http://www. mddsz.gov.si/fileadmin/mddsz.gov.si/pageuploads/dokumenti pdf/solidarity_es.pdf 
Relaciones intergeneracionales: profesoras seniors con niños y niñas del Programa Socioeducativo, Penco, Chile /Monsalve-Reyes et al..

Vasilachis de Gialdino, I. (2006) La investigación cualitativa. En Vasilachis de Gialdino, I. (Ed.) Estrategias de Investigación Cualitativa. (pp. 23-64). Barcelona: Gedisa. 